\title{
Implementation of a Spectrum Analyzer Using the Software-Defined Radio Concept
}

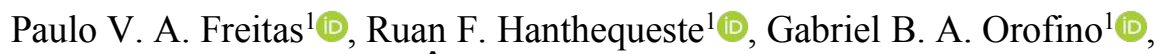

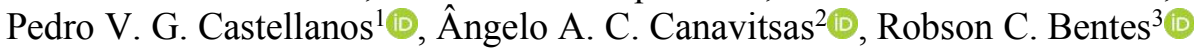 \\ ${ }^{1}$ Fluminense Federal University, Niterói, Rio de Janeiro, Brazil \\ pvafreita@gmail.com,ruanfh@id.uff.br,gabrielbach@id.uff,pcastellanos@id.uff.br \\ ${ }_{2}^{2}$ Petróleo Brasileiro S.A - Petrobras, Rio de Janeiro, RJ, Brasil \\ canavitsas@petrobras.com.br \\ ${ }^{3}$ National Telecommunications Agency - Anatel, Rio de Janeiro, RJ, Brasil \\ rbentes@anatel.gov.br
}

\begin{abstract}
The software-defined radio has been allowing engineers the possibility of implementing operations on software that were previously only possible to be performed through hardware. With the continuous increase in the processing capacity of computers, it is possible to modify in real time the operating parameters of new radios called Software Defined Radios (SDR) or use commercial devices for other purposes, in addition to those established in their design. By using a low-cost device RTL-SDR R820T2, controlled by a Raspberry Pi 3, with a free software, the present work proposes a characterization procedure in order to collect power levels measurements in a given frequency range with greater accuracy, acting as a spectrum analyzer. Preliminary measures were performed with the device embarked on a drone.
\end{abstract}

Index Terms - calibration, cognitive radio, software defined radio, spectrum analyzer.

\section{INTRODUCTION}

In recent years, with the increasing demand for services and Internet access, the need for users to be constantly connected has increased, in most cases, through mobile devices such as notebooks, tablets and smartphones. These new services are increasingly demanding frequency bands to allocate the signals transmitted to the users. However, bandwidth allocation for these services is not sufficient to ensure the correct functioning of the system. Before deploying any wireless communication system, it is necessary to conduct studies on the behavior and the availability of the signal within the scenarios in which the system will operate.

Specifically, the characterization of the radio propagation channel must be performed and, among others, the coverage area of the systems must be determined according to the parameters of each communication service. Currently, this type of study is carried out by telecommunications engineers using equipment such as the spectrum analyzer whose function, among others, is to collect the spectrum of the received signal and calculate its level at each measurement point of the scenario. Definition of coverage areas, check of interference levels and evaluation of the different propagation mechanisms can be obtained. On the other hand, this type of equipment is of high cost and, in most cases, of physical dimensions and weight that require a vehicle for its transport to, finally, enable the 
Journal of Microwaves, Optoelectronics and Electromagnetic Applications, Vol. 20, No. 4, December 2021 DOI: http://dx.doi.org/10.1590/2179-10742021v20i4254767

desired measurement campaign. In view of this, this article proposes to present the development of a prototype formed by a low-cost device RTL-SDR R820T2, controlled by a raspberry pi with a free software for measuring the power level of radiofrequency (RF) signals. With a system characterized as low cost, reduced dimensions and weight, and that facilitates or enables measurement campaigns in scenarios that, in some cases, would be impossible or very expensive with conventional equipment. An example of this type of applications and maybe the main one, is to characterize the propagation channel for an environment with Clutter. With the use of this device, it would be possible to verify the influence of natural or artificial obstacles in the propagation of the signal in personal mobile communication systems.

To develop a prototype with the characteristics mentioned above, one of the alternatives is with the use of Software-Defined Radios (SDR) technology. Currently, SDR [1] devices in conjunction with a development software allow researchers to collect information such as signal level. Initially, the values collected by the SDR devices do not have the necessary reliability for decision making, requiring a calibration of the collected values in relation to a reference value.

In the present work, we propose to use a commercial device used for digital signal reception of digital video broadcasting - Terrestrial (DVB-T) television and implement a prototype spectrum analyzer through the low-cost device: RTL-SDR R820T2 [2]. Subsequently, the prototype is calibrated to define its received power reference. The calibration stage was carried out in the propagation and antenna laboratory - LAProp - of the Fluminense Federal University (UFF). The methodology used and the calibration parameters were adjusted throughout the execution of the project. As a final part of the work, the device is embarked on a drone to perform measurements, with the objective of evaluating the degradation by diffraction caused by the sugar loaf hill, in the Urca neighborhood, in Rio de Janeiro city. The use of commercial signal reception devices to collect information such as received power is a reality thanks to software-defined radio technology. From this beginning, academics and enthusiasts all over the world have used these technologies as hobbies and in academic studies. As these devices were not manufactured for measurement activities, it is necessary a calibration as will be described ahead.

The article is organized as follows: Section II presents software-defined radio. Section III describes the SDR tools and the prototype is conceptually presented in Section IV. Section V describes the experiments carried out for system calibration. Section VI reports the use of the prototype in diffraction measurements. Finally, Section VII presents the conclusion about the present work.

\section{SOFTWARE DEFINED RADIO}

\section{A. SDR Concept}

A SDR is a wireless communication device that has part of its functions implemented by software on a computer instead of using fixed hardware components [3], [4] making it easier to integrate and set different parameters [3]-[7]. In 1999, Joseph Mitola [3] used the concept of cognition, a process of 
Journal of Microwaves, Optoelectronics and Electromagnetic Applications, Vol. 20, No. 4, December 2021 DOI: http://dx.doi.org/10.1590/2179-10742021v20i4254767

making decisions and solving problems by acquiring knowledge with past experience, to propose a new class of radios that uses spectrum more efficiently. Mitola approaches SDR as a transceiver radio whose transmitted/received signals are generated/received and modulated/demodulated by software [3], [7]. This type of implementation will allow users to change signal parameters in order to adapt it to different propagation environments and applications.

\section{B. SDR Basic Structure}

SDR, as already defined, is a communication system whose functionality is based on a combination of hardware and software operation. Figure 1 presents how an SDR device is formed, in general:

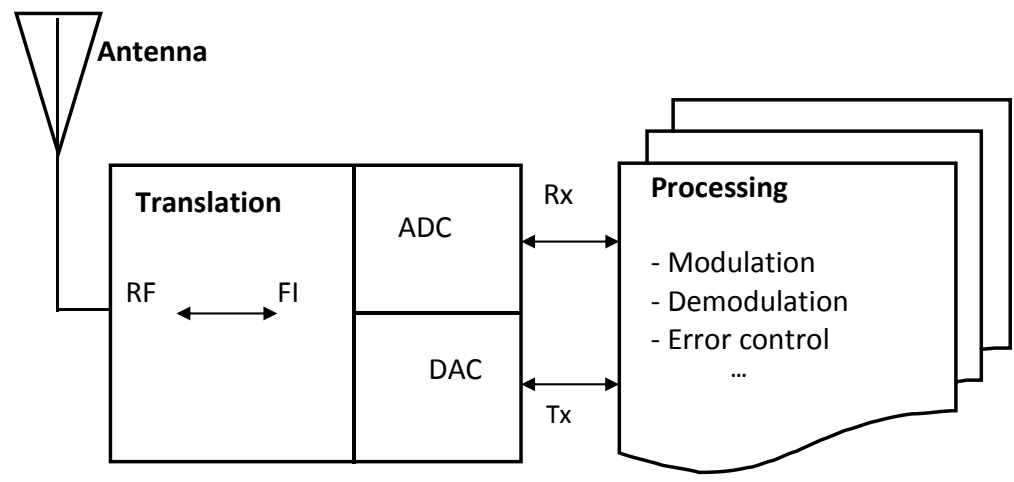

Fig. 1. Block diagram of a software-defined radio [3].

The hardware device takes care of receiving the RF signal from its antenna. The RF signal is mixed with the local oscillator frequency, and generates the intermediate frequency signal (FI). This signal is digitized by the digital analog converter (DAC) and sent to the processing unit [5], [9] which, in this work, is a computer that will process the converted signal in phase (I) and quadrature (Q).

It is important to highlight that the characteristics of the devices such as the one used have operating limitations that vary with their cost since they were not manufactured to perform RF measurements. There are options of devices in the market to be used for the same purpose, however, the low cost of the solution is very attractive. About the limitations of the device, we can mention the following: operating frequency range $(24-1766 \mathrm{MHz})$, noise floor, which depends of the used frequency and sampling rate ( 2 Msamples/s for our prototype).

\section{SDR TOOLS}

Here, the hardware components (RTL-SDR R820T2) and software (GNU-Radio) used in this work are presented. It is worth mentioning that in the literature there are some hardware and software tools that can be used in the development of SDR applications, however, those mentioned here are widely used by researchers and enthusiasts [10], [11].

\section{A. Hardware - RTL-SDR R820T2}

Among the hardware solutions for SDR models, one can identify: Universal Software Radio Peripheral (USRP), SDRplay, Airspy, FunCube Dongle Pro and RTL-SDR R820T2. In this work, due the low cost, it was used the last one: RTL-SDR R820T2. 
Journal of Microwaves, Optoelectronics and Electromagnetic Applications, Vol. 20, No. 4, December 2021 DOI: http://dx.doi.org/10.1590/2179-10742021v20i4254767

For the development of this project, the characteristics of small physical dimension and low weight allowed to embark the prototype in a drone to perform measurements.

A commercial use device for decoding radio and digital TV signals in European format (DVB-T) had its hardware configured through software to collect signal power measurements in the range of $100 \mathrm{MHz}$ to $1 \mathrm{GHz}$ [11]. As commercial application of the device is the reception of digital television signal (TVD), in the case of DVB-T, FM and Digital Audio Broadcasting (DAB) [12]. Most of these devices have nominal input impedance of $75 \mathrm{ohms}$, however, the mismatching when using $50 \mathrm{ohms}$ cables will be minimal, approximately $0.177 \mathrm{~dB}$ [13]. Figure 2 presents the device RTL-SDR R820T2.

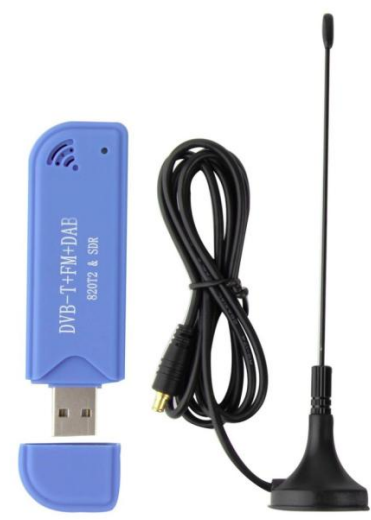

Fig. 2. Dongle R820T2.

\section{B. Software GNU Radio}

GNURadio is an open source platform or set of programs, in free software, that allows the development of signal processing components and/or processing blocks required for the implementation of SDR. The implementation can be carried out in two ways: the first using graphical tool and the second through code development in the Python language, i.e.:

- GRC (GNURadio Companion) is an open source graphical platform that allows the development of signal processing components and/or processing blocks needed for the implementation of SDR applications, using personal computers and low-cost radio equipment, having a better cost-benefit ratio for both the corporate market and the ordinary user [11], [14].

- The GNURadio platform has a series of Python codes that can be used and modified according to needs. Most processing blocks are developed from $\mathrm{C}++$ and integrated into Python code through the Simpler Wrapper and Interface Grabber (SWIG) tool. In this work, a Python code was adapted to collect the measurement of the power level received, in a certain frequency range, modifying some parameters to establish the calibration of the hardware [11], [14].

The hardware used for control, processing and storage of measurements is a Raspberry Pi 3. Figure 3 conceptually presents the prototype that is composed of the antenna, the RTL-SDR R820T2 device (Dongle RTL-SDR), the Raspberry Pi 3 hardware and a coupled power bank [15]. 
Journal of Microwaves, Optoelectronics and Electromagnetic Applications, Vol. 20, No. 4, December 2021 DOI: http://dx.doi.org/10.1590/2179-10742021v20i4254767

The measurement data collection is performed by the RTL-SDR R820T2 dongle device that tunes the RF signal of the antenna and performs analog-to-digital conversion.

An algorithm was developed to perform the necessary post-processing in the notebook that connects to the prototype through the wireless network. Data resulting from post-processing are automatically saved to files. In order for the measurement data to be quickly visualized, an algorithm was developed to generate graphs from a post-processed file [15].

IV. THE PROTOTYPE

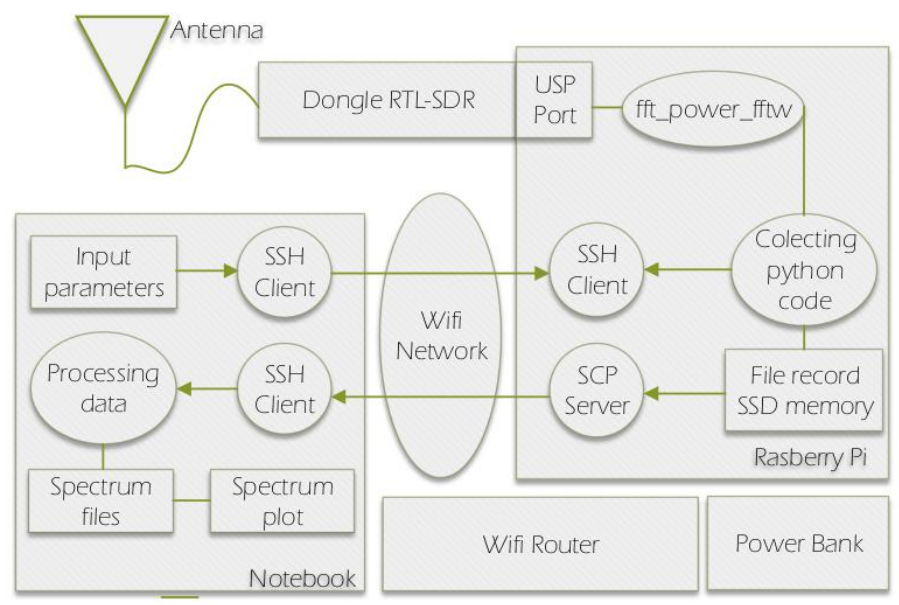

Fig. 3. Conceptual diagram of the prototype.

\section{DEVELOPMENT AND CALIBRATION}

One of the problems encountered in this type of device is the accuracy in the values of the collected data. As previously mentioned, the device used was not manufactured for the purpose of this work, which is the collection of the received signal level at various bandwidths. For this reason, the results of the measurements present linearity distortions. In order to obtain reliability in the values of the collected data, calibration and parameterization of the elements that constitute the measurement system is necessary. The calibration setup was mounted on a benchtop of the LAProp. The elements used in this calibration were an arbitrary signal generator, a power divider, two RG213 cables and a spectrum analyzer. The methodology used will be briefly described in the following sections [15].

\section{A. Cable calibration}

For the calibration of each of the elements, an Anritsu vector signal generator (MG3700A) was used to transmit a narrow band signal whose output power was kept constant at $-60 \mathrm{dBm}$. An Anritsu spectrum analyzer (MS2034A) connected through the object cable of the analysis was used at the reception. To verify the attenuation provided by the cables used in several frequencies, a scan was performed in the range of $100 \mathrm{MHz}$ to $1.7 \mathrm{GHz}$. Figure 4 shows the loss value inserted by cables 1 and 2. As expected, it is possible to observe that the attenuation value varies, with a maximum attenuation of $2.9 \mathrm{~dB}$ on cable 2 , and $2.5 \mathrm{~dB}$ on cable 1 , at frequencies of $1500 \mathrm{MHz}$ and $1700 \mathrm{MHz}$, 
respectively [14].

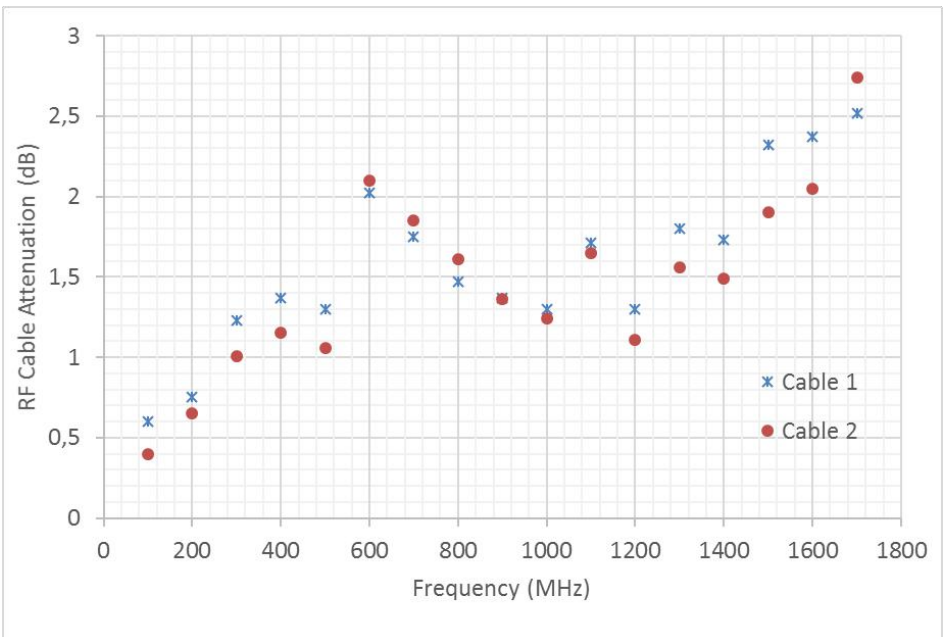

Fig. 4. Cable calibration.

\section{B. Prototype Calibration}

After the calibration of the cables and the power divider ( $3 \mathrm{~dB}$ in each branch), the device for measuring was calibrated. The schematic diagram of interconnections for the calibration of the RTLSDR R820T2 is in Fig.5. The following elements were used: vector signal generator (Anritsu MG3700A), spectrum analyzer (Anritsu MS2034A), calibrated cables, signal divider, N-SMA connector adapter, notebook, RTL SDR R820T2, and "rtl_power.c" code that controls the RTLSDR and collects the signal power [15], [16].

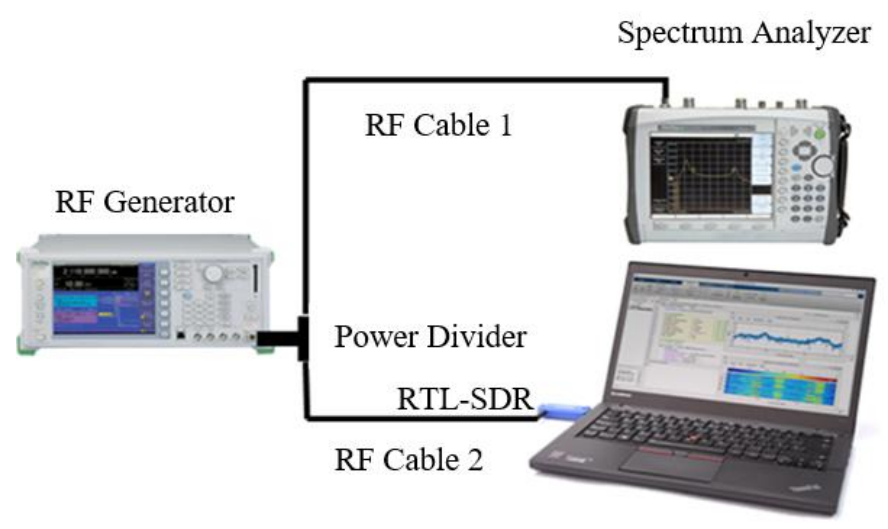

Fig. 5. Schematic diagram of interconnections for the prototype calibration.

For calibration, a continuous wave $(\mathrm{CW})$ signal test was transmitted using a signal generator (MG3700A) with a signal power output of $-60 \mathrm{dBm}$. This signal was divided using a power divider that added 3dB loss in each branch. The spectrum analyzer and the SDR device (RTL-SDR R820T2) were connected via cables 1 and 2, respectively, to each branch of the divider power for the collection of received signal levels. The RTL-SDR R820T2 was plugged into a notebook via USB port. Figure 6 presents the assembly of the test setup on the bench of the UFF propagation laboratory [15]. For the calibration step, the spectrum analyzer was configured with span of $8 \mathrm{MHz}$, resolution bandwidth of 3 $\mathrm{kHz}$, visual bandwidth of $30 \mathrm{kHz}$ and sample detector in average mode. For the calibration measurements about 200 samples were used to calculate the received power.

Brazilian Microwave and Optoelectronics Society-SBMO received 31 Jan 2021; for review 31 Jan 2021; accepted 15 May 2021 
Journal of Microwaves, Optoelectronics and Electromagnetic Applications, Vol. 20, No. 4, December 2021 DOI: http://dx.doi.org/10.1590/2179-10742021v20i4254767

After accounting for all losses entered by the setup elements, calibration was performed by comparing the power level values measured in the spectrum analyzer and the values measured through the software that collects the data via RTL-SDR R820T2, taking into account the settings adjusted through the code rtl_power.c.

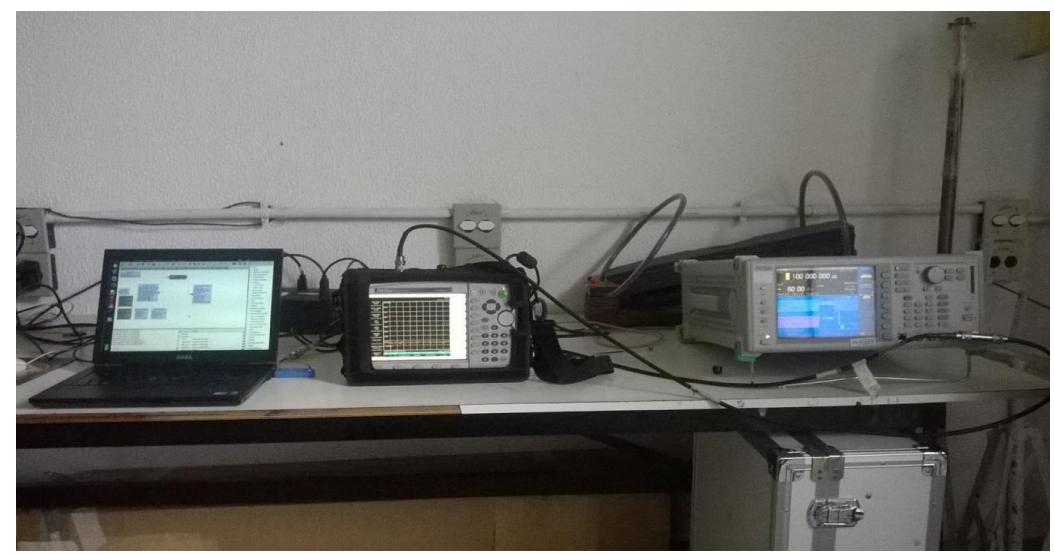

Fig. 6. Mounting for calibration of the RTL-SDR R820T2.

The RTL-SDR R820T2 has parameters that can be configured in real time. The most important for device calibration is the gain. The device allows configure eighteen values of gain for reception. However, as observed in the experiments, the gains defined by the manufacturer do not correspond to the measured values, which corroborates the need of the calibration process. Fig. 7 shows the measured received signal strength indicator in $\mathrm{dB}$ (RSSI) versus frequency for the gains of $0 \mathrm{~dB}, 8 \mathrm{~dB}$, $10 \mathrm{~dB}$ and $12 \mathrm{~dB}$ configured on the device, related to the same input. In this figure it is possible to observe that the difference between the gain of $0 \mathrm{~dB}$ and $8 \mathrm{~dB}$, at $200 \mathrm{MHz}$, is approximately $12 \mathrm{~dB}$. If we observe the same difference, but this time at the frequency of $500 \mathrm{MHz}$, the value is approximately $11 \mathrm{~dB}[15]$.

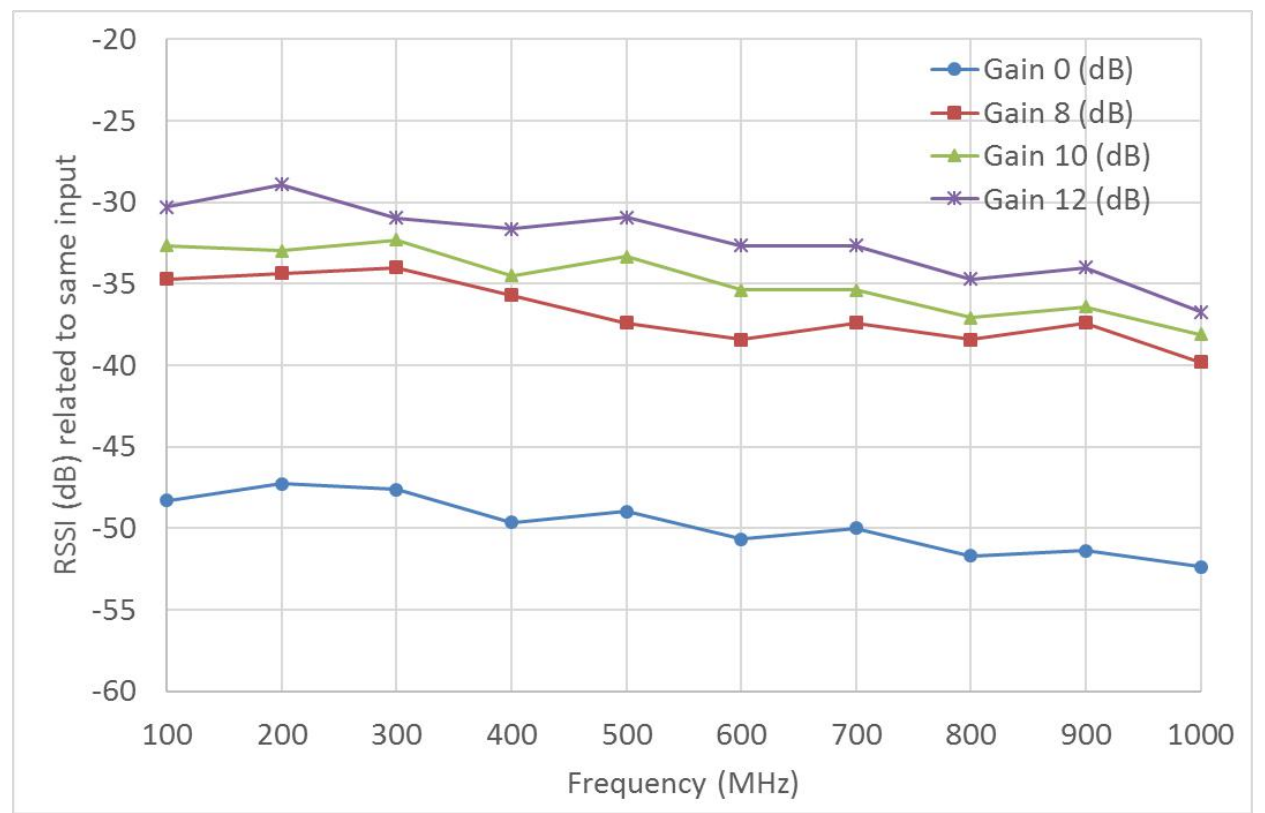

Fig. 7. Power level variation received by RTL-SDR R820T2 in different gains.

After defining the actual gain values in each frequency, we compared the RSSI values collected by 
Journal of Microwaves, Optoelectronics and Electromagnetic Applications, Vol. 20, No. 4, December 2021 DOI: http://dx.doi.org/10.1590/2179-10742021v20i4254767

the RTL-SDR R820T2 to those measured by the spectrum analyzer in $\mathrm{dBm}$. By balancing the losses (Fig. 7), it is verified that the power signal level received in the spectrum analyzer is $-66 \mathrm{dBm} \pm 1 \mathrm{~dB}$, on average, at the frequency of $500 \mathrm{MHz}$ [15]. For the other frequencies, the power levels received depend on the attenuation values added by the cables like shown in Fig. 4. Analogous to the previous section, Table I displays the correction value that should be applied to the reading obtained through the device, depending on the gain configuration $(0$ or $8 \mathrm{~dB})$ [15]. The third column displays the value obtained in the spectrum analyzer.

TABLE I. CORRECTION VALUE ADDED TO RTL-SDR R820T2 FOR 0 DB AND 8 DB GAINS

\begin{tabular}{cccc}
\hline $\begin{array}{c}\text { Frequency } \\
\text { (MHz) }\end{array}$ & $\begin{array}{c}\text { RTL-SDR R820T2: Calibration value } \\
\text { Gain 0 dB } \\
\text { (dB) }\end{array}$ & $\begin{array}{c}\text { Anritsu MS2034A } \\
\text { Gain 8 dB } \\
\text { (dB) }\end{array}$ & $\begin{array}{c}\text { (dBm) } \\
\text { (MB) }\end{array}$ \\
\hline 100 & $-14,03$ & $-0,06$ & $-64,78$ \\
200 & $-13,38$ & 0,96 & $-63,33$ \\
300 & $-17,95$ & $-1,87$ & $-65,45$ \\
400 & $-16,77$ & $-2,48$ & $-63,22$ \\
500 & $-14,65$ & $-0,6$ & $-66,22$ \\
600 & $-16,04$ & $-3,21$ & $-63,7$ \\
700 & $-20,49$ & $-5,32$ & $-65,74$ \\
800 & $-20,48$ & $-6,96$ & $-64,3$ \\
900 & $-17,18$ & $-3,42$ & $-65,04$ \\
1000 & $-17,80$ & $-4,78$ & $-65,82$ \\
\hline
\end{tabular}

After calibrating the prototype, a $6 \mathrm{MHz}$ band signal test was transmitted and then compared in both equipment, the prototype and the spectrum analyzer. Figure 8 shows a measurement performed on the digital channel 29 (central frequency $563.143 \mathrm{MHz}$ ). In this figure, the measurement performed by the calibrated prototype is compared to the one performed by the spectrum analyzer. A good accordance of values in the measured band is observed, except for the noise floor measured in the prototype, due to manufacture characteristics.

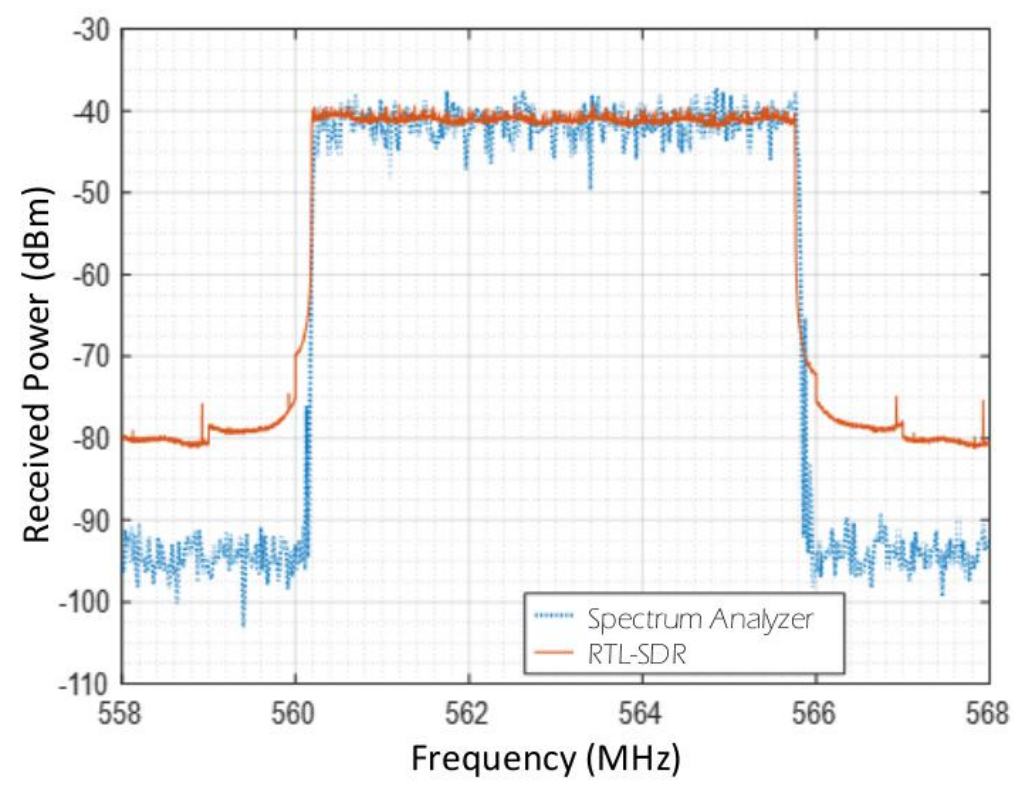

Fig. 8. Measurements of channel 29 UHF (563.143 MHz).

Brazilian Microwave and Optoelectronics Society-SBMO received 31 Jan 2021; for review 31 Jan 2021; accepted 15 May 2021 
Journal of Microwaves, Optoelectronics and Electromagnetic Applications, Vol. 20, No. 4, December 2021 DOI: http://dx.doi.org/10.1590/2179-10742021v20i4254767

\section{DIFFRACTION MEASUREMENTS}

Studies are being conducted to characterize the additional attenuation due to diffraction. One of the places chosen to study this type of attenuation was the Urca hill, in Rio de Janeiro city. In the vicinity of this hill it is possible to get sight to the transmitting antenna, located on the hill of Sumaré, both showed in Fig. 9, respectively, on the left and right. However, in certain other positions, it is possible to observe the existence of obstruction of the target line to the same transmitting antenna. Hence, the signal received in this position is degraded by the phenomenon of diffraction. The measurements for analyzing the attenuation caused by this phenomenon using conventional equipment, given the characteristics of dimensions and weight, could only be carried out at ground level.

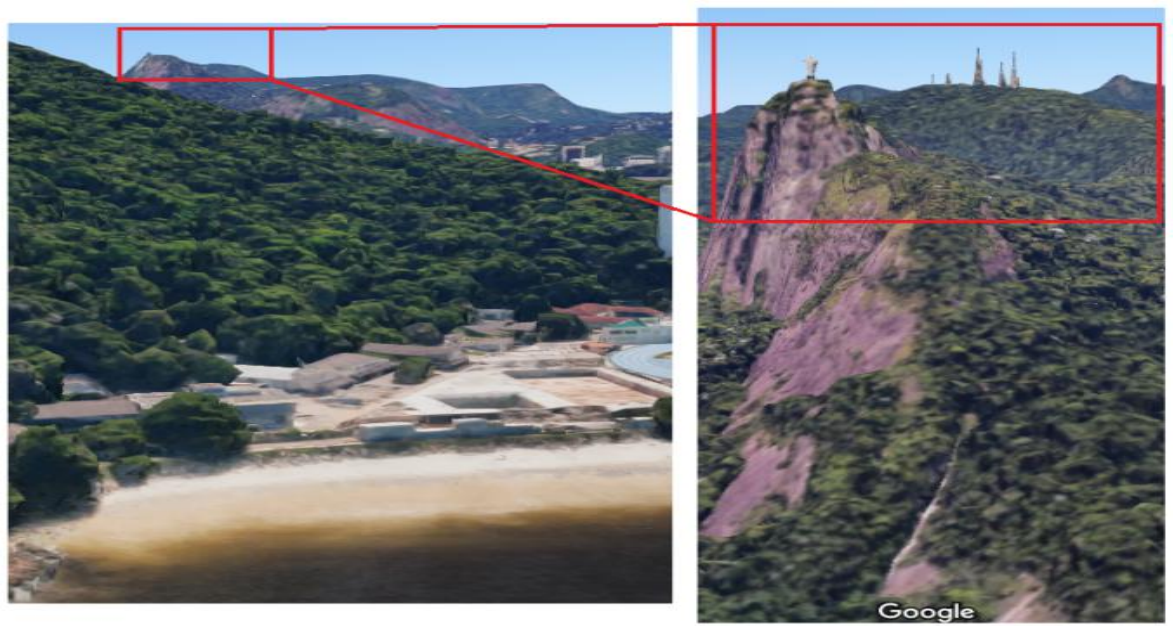

Fig. 9. Transmitting antenna sight.

With the aim of testing the prototype, a preliminary campaign of measurements was carried out. As a first stage, measures were collected at ground level, with the prototype developed and with a spectrum analyzer. In order to perform the measurements at different altitudes, the prototype developed in this work was encapsulated using acrylic material to give the device physical protection and lower total weight to the set: 500 grams. Subsequently, the prototype and antenna were coupled to a DJI Inspire I drone as seen in Fig. 10.

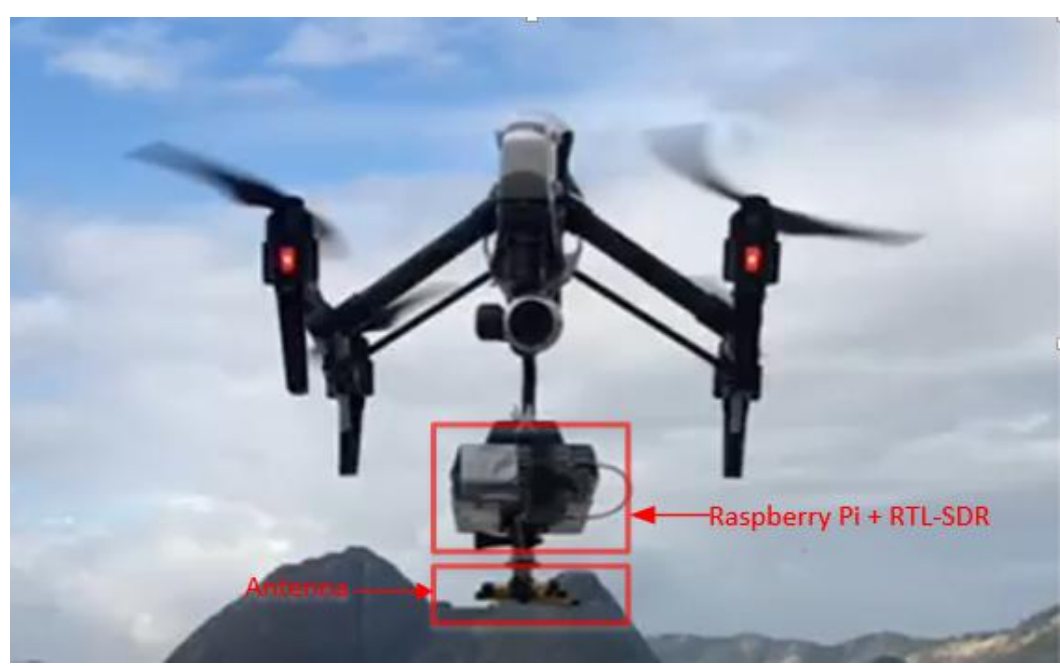

Fig. 10. Measurement prototype coupled to drone. 


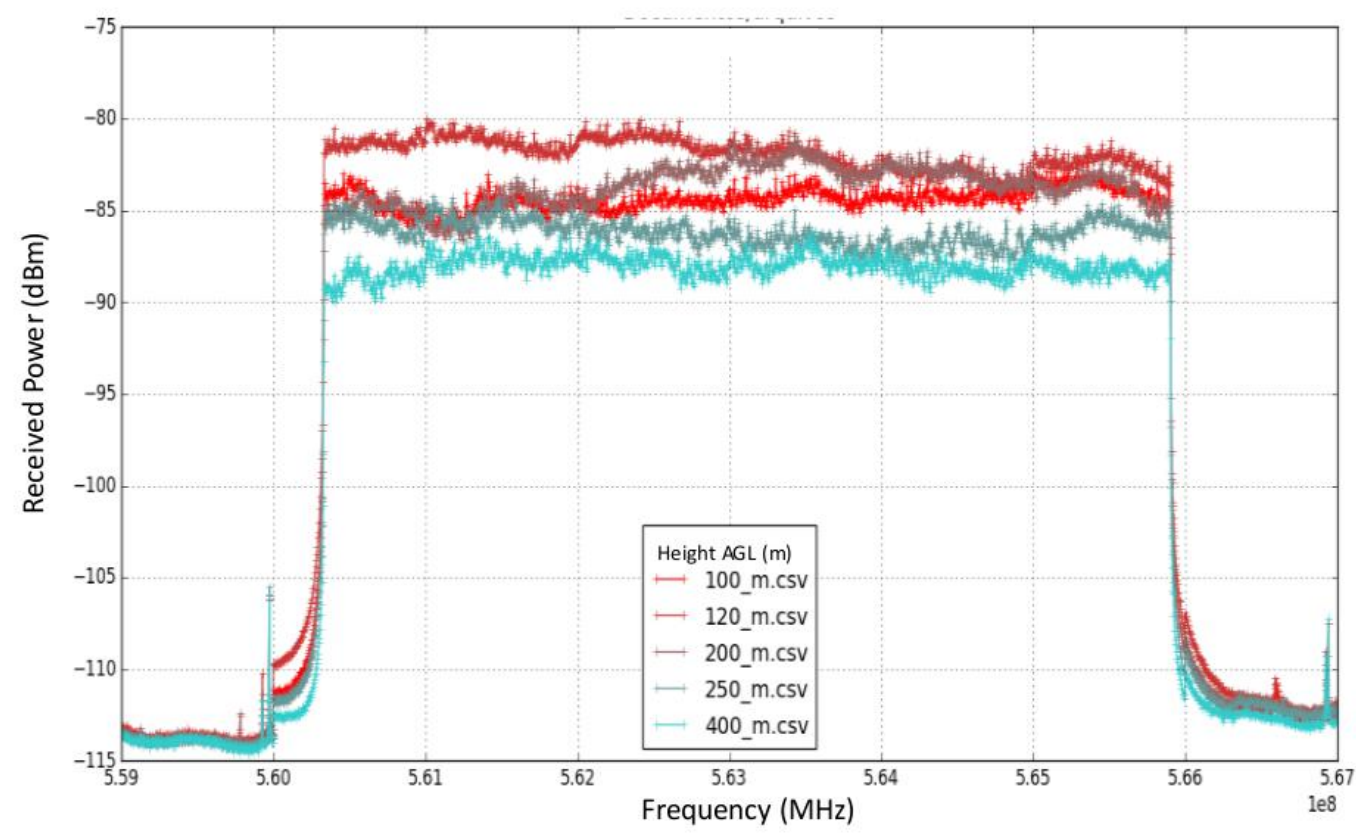

Fig. 11. Measured power levels in different distances.

A series of measurements at different altitudes and propagation conditions were performed in order to compare the additional attenuation due to diffraction, and Fig. 11 presents the overlap of measurements performed with the prototype already calibrated. It is possible to observe that at the altitude of 120 meters the level of the power received by frequency is higher than for the other altitudes, possibly indicating to be a peak of irradiation of the transmitting antenna [15]. It should be noted that the result of altitude measures is not the goal of work. To make a comparison with other jobs, a greater number of measures must be performed.

\section{CONCLUSION}

In this work, a prototype was developed for spectrum measurement using low-cost hardware and free software. The capabilities and characteristics of software-defined radio development tools were studied. The RTL-SDR R820T2, Raspberry Pi 3 and GNURadio/Python/C platform were used to perform calibration and implementation of a spectrum analyzer on a low-cost device, reduced physical dimensions, and weight.

Before performing the experiments, the respective calibrations of the components were performed for the assembled setup, including the RTL-SDR R820T2, because it is a device not designed to perform RF measurements. In the field, the measurements were performed by coupling the prototype to a drone, with the objective of characterizing the additional attenuation due to the diffraction in the vicinity of the Urca hill, in the city of Rio de Janeiro. In measurements performed comparing the values measured by the spectrum analyzer and the values measured and adjusted by the prototype (both equipment at ground level) it was possible to verify that the results are promising.

references 
Journal of Microwaves, Optoelectronics and Electromagnetic Applications, Vol. 20, No. 4, December 2021

DOI: http://dx.doi.org/10.1590/2179-10742021v20i4254767

[1] A. Fanan, N. Riley, M. Mehdawi, M. Ammar and M. Zolfaghari, "Comparison of spectrum occupancy measurements using software defined radio RTL-SDR with a conventional spectrum analyzer approach", 2015 23rd Telecommunications Forum Telfor (TELFOR), Belgrade, Serbia, 2015, pp. 200-203, doi: 10.1109/TELFOR.2015.7377447.

[2] M. Abirami, V. Hariharan, M. B. Sruthi, R. Gandhiraj and K. P. Soman, "Exploiting GNU radio and USRP: An economical test bed for real time communication systems", 2013 Fourth International Conference on Computing, Communications and Networking Technologies (ICCCNT), Tiruchengode, India, 2013, pp. 1-6, doi: 10.1109/ICCCNT.2013.6726630.

[3] J. Mitola, "Cognitive radio for flexible mobile multimedia communications", Mobile Multimedia Communications, (MoMuC'99) 1999 IEEE International Workshop, pp. 3-10, 1999.

[4] X. Hong, J. Wang, C. X. and J. Shi, "Cognitive radio in 5G: a perspective on energy-spectral efficiency trade-off", IEEE Communications Magazine, vol. 52, pp. 46-53, 2014.

[5] R. Hosking, "Software Defined Radio Handbook", Pentek, Inc., 2011.

[6] S. Haykin, "Cognitive radio: brain-empowered wireless communications", IEEE Journal Selected Areas in Communications, vol. 23, no. 2, pp. 201-220, 2005.

[7] J. Mitola, "The software radio architecture", IEEE Communications Magazine, 1995, vol. 33, pp. 26-38.

[8] Ettus Research LLC. http://www.ettus.com.

[9] http://www.wirelessinnovation.org

[10] http://datasheetcafe.databank.netdna-cdn.com/wp-content/uploads/2015/09/RTL2832U.pdf

[11] K. Ranous.“ RTL-SDR for Linux Quick Start Guide”, 3rd. Ed., 2020.

[12] D. Pajuelo, Y. Iano, P. E. R. Cardoso, F. C. Cabello, J. Leon, R. O. Barbieri, D. Izario and B. Izario, "Technical Study on the Transmission of HDR Content over a Broadcast Channel", SET Journal of Broadcasting, pp. 29-35.

[13] C. Laufer, The Hobbyist's Guide to the RTL-SDR: Really Cheap Software Defined Radio 1st Edition.

[14] L. G. Barros, "O rádio definido por software", Brasília, 2007. Course Completion Work - Graduation in Electrical Engineering - University of Brasília.

[15] R. F. Hanthequeste, "Sistema de sensoriamento espectral para uso em campo utilizando software livre e Raspberry pi", Niterói, 2019. Course Completion Work - Graduation in Telecommunications Engineering - Fluminense Federal University.

[16] Repository containing the project rtl-power-fftw. https://github.com/AD-Vega/rtl-power-fftw. 